\title{
Impact of goal-directed hemodynamic management on the incidence of acute kidney injury in patients undergoing partial nephrectomy: a pilot randomized controlled trial
}

Qiong-Fang $\mathrm{Wu}^{1+}$, Hao Kong ${ }^{1 \dagger}$, Zhen-Zhen $\mathrm{Xu}^{1}$, Huai-Jin $\mathrm{Li}^{1}$, Dong-Liang $\mathrm{Mu}^{1}$ and Dong-Xin Wang ${ }^{1,2^{*}}$ (D)

\begin{abstract}
Background: The incidence of acute kidney injury (AKI) remains high after partial nephrectomy. Ischemiareperfusion injury produced by renal hilum clamping during surgery might have contributed to the development of AKI. In this study we tested the hypothesis that goal-directed fluid and blood pressure management may reduce AKI in patients following partial nephrectomy.

Methods: This was a pilot randomized controlled trial. Adult patients who were scheduled to undergo partial nephrectomy were randomized into two groups. In the intervention group, goal-directed hemodynamic management was performed from renal hilum clamping until end of surgery; the target was to maintain stroke volume variation $<6 \%$, cardiac index $3.0-4.0 \mathrm{~L} / \mathrm{min} / \mathrm{m}^{2}$ and mean arterial pressure $>95 \mathrm{mmHg}$ with crystalloid fluids and infusion of dobutamine and/or norepinephrine. In the control group, hemodynamic management was performed according to routine practice. The primary outcome was the incidence of AKI within the first 3 postoperative days.
\end{abstract}

Results: From June 2016 to January 2017, 144 patients were enrolled and randomized (intervention group, $n=72$; control group, $n=72$ ). AKI developed in $12.5 \%$ of patients in the intervention group and in $20.8 \%$ of patients in the control group; the relative reduction of AKI was $39.9 \%$ in the intervention group but the difference was not statistically significant (relative risk $0.60,95 \%$ confidence interval $[\mathrm{CI}] 0.28-1.28 ; P=0.180$ ). No significant differences were found regarding AKI classification, change of estimated glomerular filtration rate over time, incidence of postoperative 30-day complications, postoperative length of hospital stay, as well as 30-day and 6-month mortality between the two groups.

(Continued on next page)

\footnotetext{
* Correspondence: dxwang65@bjmu.edu.cn; wangdongxin@hotmail.com

${ }^{\dagger}$ Qiong-Fang Wu and Hao Kong contributed equally to this work.

'Department of Anesthesiology and Critical Care Medicine, Peking University First Hospital, Beijing 100034, China

${ }^{2}$ Outcomes Research Consortium, Cleveland, OH, USA
} 
(Continued from previous page)

Conclusion: For patients undergoing partial nephrectomy, goal-directed circulatory management during surgery reduced postoperative AKI by about 40\%, although not significantly so. The trial was underpowered. Large sample size randomized trials are needed to confirm our results.

Trial registration: Clinicaltrials.gov identifier: NCT02803372. Date of registration: June 6, 2016.

Keywords: Partial nephrectomy, Hemodynamic management, Acute kidney injury

\section{Background}

Partial nephrectomy through an open incision or laparoscopic way is increasingly used to treat renal tumor with benefits of sparing nephrons and preserving renal function [1]. However, removal of renal parenchyma [2, 3], suture damage and ischemia-reperfusion injury [4] during partial nephrectomy all compromise renal function. The reported incidence of acute kidney injury (AKI) after partial nephrectomy ranged from 16.5 to $42 \%$, and even up to $54 \%$ in solitary kidney patients [5-7]. In the study of Rajan et al. [8], 39\% of patients developed AKI after partial nephrectomy; specifically, 33\% had stage 1, $4 \%$ had stage 2 , and $2 \%$ had stage 3 AKI after surgery. The occurrence of postoperative AKI is significantly associated with increased risks of renal function decline and chronic kidney diseases [6, 9], as well as adverse cardiovascular events and even mortality [10].

Many effects have been performed to preserve residual renal function, such as improving surgical skill, sparing more normal nephrons and shortening ischemic duration. Furthermore, improving renal tissue perfusion and alleviating ischemia-reperfusion injury (IRI) caused by renal hilum clamping may also provide renal protection. Indeed, stroke volume guided fluid infusion and inotropic therapy improves global oxygen delivery, microvascular flow and tissue oxygenation [11]. However, evidence regarding the effect of circulatory management on AKI development after partial nephrectomy is limited. Available studies mainly focused on major abdominal surgeries and gave conflicting results. For example, Pearse et al. [12] found that cardiac output-guided hemodynamic management did not reduce complications; whereas Futier et al. [13] reported that individualized blood pressure management reduced postoperative organ dysfunction. Partial nephrectomy usually involves renal hilum clamping and declamping, similar to kidney transplantation to some extent. Kidney transplantation represents a typical situation of ischemia-reperfusion; it is recommended to maintain high central venous pressure $(\mathrm{CVP}>8 \mathrm{mmHg})$ and high mean arterial pressure (MAP > $95 \mathrm{mmHg}$ ) with crystalloid hydration and vasoactive drugs at the time of hilum declamping in order to improve reperfusion [14-18]. As a dynamic parameter, stroke volume variation can be used to replace central venous pressure in evaluating volume status [19]. We hypothesized that a similar strategy of crystalloid hydration and hemodynamic management based on stroke volume monitoring during partial nephrectomy might also protect kidney.

The purpose of this pilot randomized trial was to test the effects of goal-directed fluid and blood pressure management on the incidence of AKI in patients following partial nephrectomy for renal cancer.

\section{Methods \\ Study design}

This pilot randomized controlled trial which was performed in a tertiary hospital in Beijing, China. The study protocol was approved by the Clinical Research Ethics Committee of Peking University First Hospital (2016[1118]) and was a priori registered with ClinicalTrials.gov (NCT02803372) on June 6, 2016. Written informed consents were obtained from all participants.

\section{Participants}

Potential participants were screened the day before surgery. The inclusion criteria were adult ( $\geq 18$ years) patients scheduled to undergo elective laparoscopic or open partial nephrectomy. Patients who met any of the following criteria were excluded: (1) severe renal function impairment (estimated glomerular filtration rate [eGFR] $<45 \mathrm{ml} / \mathrm{min} / 1.73 \mathrm{~m}^{2}$ ), (2) arrhythmia or impaired cardiac function (New-York Heart Association classification $\geq$ III), (3) bilateral renal surgery, (4) solitary kidney, (5) anticipated massive blood loss $(\geq 800 \mathrm{ml}$ ) and requirement of artificial colloid infusion, or (6) American Society Anesthesiologist classification $\geq$ IV.

Baseline data were collected after obtaining written informed consents and included demographic variables, previous comorbidities, results of important laboratory tests, results of tumor examination, American Society of Anesthesiologists classification, and Preoperative Aspects and Dimensions Used for an Anatomical (PADUA) score. PADUA score is a simple anatomical system used to predict the risk of perioperative surgical and medical complications in patients undergoing nephron-sparing surgery; the score ranges from 6 to 14 , a score $\geq 8$ indicates high risk of complications [20]. 


\section{Randomization and blinding}

Patients were randomly allocated to either the intervention group (goal-directed hemodynamic management) or the control group (routine hemodynamic management) in a 1:1 ratio according to computer-generated random numbers. The allocation was sealed in opaque envelopes until shortly before anesthesia induction. Randomization and group assignment were performed by a study coordinator who did not participate in perioperative care and data collection. Anesthesiologists who were responsible for anesthetic management were not involved in followup. Investigators who performed postoperative follow-up and patients were masked from study group assignment.

\section{Intervention, anesthesia and perioperative care}

Routine intraoperative monitoring included electrocardiogram, non-invasive blood pressure, pulse oxygen saturation, end-tidal carbon dioxide, volatile anesthetic concentration, bispectral index, and urine output. Invasive blood pressure was monitored after anesthesia induction. Intraoperative blood pressure and heart rate were recorded automatically every $10 \mathrm{~s}$ by the Anesthesia Information System. Patients in the intervention group were connected to a LiDCO ${ }^{\text {rapid }}$ monitor (LiDCO Ltd., HM81-01, UK) which continuously displayed hemodynamic variables including stroke volume variation (SVV) and cardiac index (CI).

No premedication was administered. General anesthesia was performed for all patients. Anesthesia was induced with midazolam, propofol/etomidate, sufentanil, and rocuronium; and maintained with intravenous propofol, remifentanil/sufentanil, rocuronium/cisatracurium, and $50 \%$ nitrous oxide inhalation. The target was to maintain bispectral index between 40 and 60. Patients were ventilated through an endotracheal tube or a laryngeal mask airway with a tidal volume of $8-10 \mathrm{ml} / \mathrm{kg}$.

For patients in the intervention group, goal-directed hemodynamic management was performed from renal hilum clamping until end of surgery. The target was to maintain a $\mathrm{SVV}<6 \%$, a CI between 3.0 and $4.0 \mathrm{~L} / \mathrm{min} /$ $\mathrm{m}^{2}$, and a MAP $>95 \mathrm{mmHg}$ with crystalloid fluids and intravenous infusion of dobutamine and/or norepinephrine. Volume loading with $250-\mathrm{ml}$ crystalloid fluid was rapidly infused to achieve $\mathrm{SVV}<6 \%$. Dobutamine was infused from $2 \mu \mathrm{g} / \mathrm{kg} / \mathrm{min}$ and adjusted by anesthesiologists to achieve the target of $\mathrm{CI}$ and MAP. Ephedrine

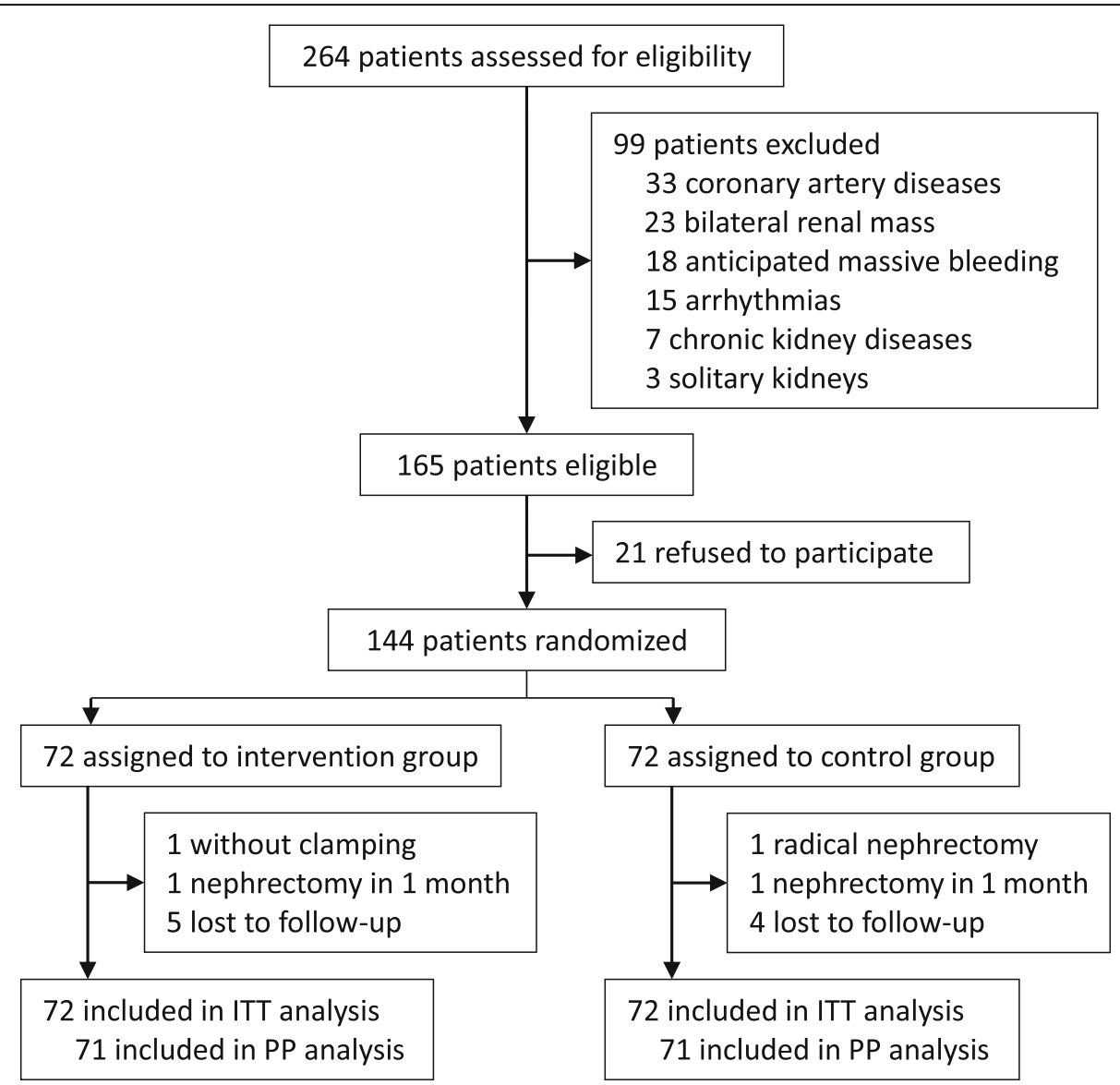

Fig. 1 Flowchart of the study. ITT, intention-to treat. PP, per-protocol 
was also administered to achieve this target when necessary. In case that hemodynamic target was not achieved or heart rate $>120 \%$ of baseline or $>100$ beats per minute, norepinephrine infusion was added and adjusted. For patients in the control group, hemodynamic management was performed according to routine practice, i.e., blood pressure was maintained within $20 \%$ from baseline and a urine output $>0.5 \mathrm{ml} / \mathrm{kg} / \mathrm{h}$ with crystalloid fluids and intravenous injection of ephedrine.

Laparoscopic partial nephrectomy was performed through retropneumoperitoneum with a carbon dioxide pressure of 12-14 mmHg. Open partial nephrectomy was performed when the laparoscopic way was not applicable. Surgery was performed with patients in the lateral position or, in some cases, in the supine position. As a routine practice, renal artery clamping was applied during resection of renal parenchyma. Diuretics such as mannitol and/or furosemide were administered at the discretion of attending surgeons. Dexamethasone (5-10 $\mathrm{mg}$ ) and tropisetron $(5 \mathrm{mg})$ could be administered to prevent postoperative nausea and vomiting. Non-steroid anti-inflammatory drugs and artificial colloids were not allowed in both groups. Blood transfusion was provided when considered necessary.

After surgery, analgesia were provided with a patientcontrolled analgesia pump which was established with

Table 1 Baseline data

\begin{tabular}{|c|c|c|c|}
\hline & Intervention group $(n=72)$ & Control group $(n=72)$ & ASD \\
\hline Age (years) & $54.1 \pm 12.1$ & $54.5 \pm 12.3$ & 0.028 \\
\hline Male gender & $48(66.7 \%)$ & $43(59.7 \%)$ & 0.144 \\
\hline Body Mass Index $\left(\mathrm{kg} / \mathrm{m}^{2}\right)$ & $25.2 \pm 3.3$ & $25.0 \pm 3.4$ & 0.040 \\
\hline \multicolumn{4}{|l|}{ Comorbidity } \\
\hline Hypertension & $26(36.1 \%)$ & $34(47.2 \%)$ & 0.227 \\
\hline Diabetes & $13(18.1 \%)$ & $12(16.7 \%)$ & 0.037 \\
\hline Respiratory diseases ${ }^{a}$ & $2(2.8 \%)$ & $7(9.7 \%)$ & 0.290 \\
\hline Others $^{\mathrm{b}}$ & $6(8.3 \%)$ & $5(6.9 \%)$ & 0.052 \\
\hline Hemoglobin (g/L) & $144.1 \pm 15.2$ & $142.8 \pm 14.8$ & 0.086 \\
\hline Albumin (g/L) & $44.9 \pm 3.5$ & $44.3 \pm 4.2$ & 0.153 \\
\hline $\mathrm{eGFR}\left(\mathrm{mL} / \mathrm{min} / 1.73 \mathrm{~m}^{2}\right)^{c}$ & $83 \pm 18$ & $84 \pm 14$ & 0.134 \\
\hline Baseline SBP $(\mathrm{mmHg})^{d}$ & $132 \pm 17$ & $139 \pm 19$ & 0.397 \\
\hline Baseline MAP $(\mathrm{mmHg})^{\mathrm{d}}$ & $96 \pm 13$ & $99 \pm 12$ & 0.228 \\
\hline Baseline HR (bpm) ${ }^{d}$ & $73 \pm 9$ & $72 \pm 10$ & 0.084 \\
\hline ASA classification & & & 0.057 \\
\hline 1 & $32(44.4 \%)$ & $30(41.7 \%)$ & \\
\hline 2 & $36(50.0 \%)$ & $38(52.8 \%)$ & \\
\hline 3 & $4(5.6 \%)$ & $4(5.6 \%)$ & \\
\hline Maximal mass diameter $(\mathrm{cm})$ & $2.8(2.2,4.0)$ & $3(2.1,4.2)$ & 0.109 \\
\hline PADUA score ${ }^{e}$ & $7.5 \pm 1.1$ & $7.5 \pm 1.0$ & 0.013 \\
\hline Histology of cancer ${ }^{f}$ & & & 0.092 \\
\hline Clear cell carcinoma & 49 (68.1\%) & $50(69.4 \%)$ & \\
\hline Angiomyolipoma & $15(20.8 \%)$ & $9(12.5 \%)$ & \\
\hline Others $^{g}$ & $8(11.1 \%)$ & $13(18.1 \%)$ & \\
\hline
\end{tabular}

Data are mean $\pm S D$, number (\%), or median (interquartile range)

ASD absolute standardized difference (an ASD of $\geq 0.327$ is considered imbalanced between the two groups), eGFR estimated glomerular filtration rate, SBP systolic blood pressure, MAP mean arterial pressure, HR heart rate, PADUA Preoperative Aspects and Dimensions Used for an Anatomical scores

${ }^{a}$ Included asthma (2 patients), chronic obstructive pulmonary disease (1 patient), obstructive sleep apnea (3 patients), history with lobectomy ( 2 patients), metastatic lung cancer (1 patient)

${ }^{\mathrm{b}}$ Included coronary atherosclerotic heart disease (3 patients), gout (3 patients), lower limb artery thrombosis (1 patient), vasovagal syncope (1 patient), and liver disease (3 patients)

${ }^{c}$ Calculated according to the Chronic Kidney Disease Epidemiology Collaboration (CKD-EPI) equation [22]

${ }^{\mathrm{d}}$ Measured in the operating room before anesthesia induction

ePADUA scores, a simple anatomical system used to predict the risk of perioperative surgical and medical complications in patients undergoing nephron-sparing surgery. The score ranges from 6 to 14 , a score $\geq 8$ indicates higher risk of complications [20]

${ }^{\mathrm{f}}$ According to pathological examination after surgery

Included chromophobe cell carcinoma ( 2 patients), papillary carcinoma (4 patients), malignant epithelial tumor ( 2 patients), cystic lesions (4 patients), oncocytic carcinoma ( 3 patients), unclassified renal cell carcinoma (5 patients), and inflammatory lesion ( 1 patient) 
Table 2 Intra-/postoperative variables

\begin{tabular}{|c|c|c|c|}
\hline & Intervention group $(n=72)$ & Control group $(n=72)$ & $P$ value \\
\hline Airway management & & & $>0.999$ \\
\hline Endotracheal tube & 59 (81.9\%) & 59 (81.9\%) & \\
\hline Laryngeal mask & $13(18.1 \%)$ & $13(18.1 \%)$ & \\
\hline \multicolumn{4}{|l|}{ Intraoperative anesthetics } \\
\hline Use of midazolam & $18(25 \%)$ & $21(29.2 \%)$ & 0.574 \\
\hline Use of etomidate & $30(41.7 \%)$ & $35(48.6 \%)$ & 0.402 \\
\hline Use of cisatracurium & $50(69.4 \%)$ & $47(65.3 \%)$ & 0.594 \\
\hline Propofol (mg) & $537(393,686)$ & $510(365,650)$ & 0.562 \\
\hline Sufentanil $(\mu \mathrm{g})$ & $30(20,38)$ & $25(20,39)$ & 0.689 \\
\hline Remifentanil $(\mu \mathrm{g})$ & $560(0,837)$ & $562(30,815)$ & 0.781 \\
\hline Rocuronium (mg) & $50(40,50)$ & $50(40,50)$ & 0.998 \\
\hline \multicolumn{4}{|l|}{ Vasoactive drugs } \\
\hline Use of dobutamine & $65(90.3 \%)$ & $0(0.0 \%)$ & $<0.001$ \\
\hline Use of norepinephrine & $6(8.3 \%)$ & $0(0.0 \%)$ & 0.028 \\
\hline Use of ephedrine & $28(38.9 \%)$ & $23(31.9 \%)$ & 0.384 \\
\hline Use of diuretics during surgery & $54(75.0 \%)$ & $56(77.8 \%)$ & 0.695 \\
\hline Mannitol (g) & $25(25,25)(n=39)$ & $25(25,25)(n=37)$ & 0.201 \\
\hline Furosemide (mg) & $10(10,10)(n=49)$ & $10(10,10)(n=52)$ & 0.215 \\
\hline Dexamethasone (mg) & $5(5,5)$ & $5(5,5)$ & 0.229 \\
\hline Fluids infusion (ml) ${ }^{a}$ & $1650(1525,2100)$ & $1600(1213,1800)$ & 0.012 \\
\hline Urine output (ml) & $400(200,600)$ & $300(200,488)$ & 0.020 \\
\hline Estimated blood loss (ml) & $50(30,100)$ & $50(23,100)$ & 0.885 \\
\hline Packed red blood cells & $1(1.4 \%)$ & $1(1.4 \%)$ & $>0.999$ \\
\hline Duration of anesthesia (min) & $131 \pm 42$ & $135 \pm 49$ & 0.649 \\
\hline Type of surgery & & & $>0.999$ \\
\hline Laparoscopic & $64(88.9 \%)$ & $64(88.9 \%)$ & \\
\hline Open-abdominal & $8(11.1 \%)$ & $8(11.1 \%)$ & \\
\hline Surgical position & & & $>0.999$ \\
\hline Supine & $3(4.2 \%)$ & $3(4.2 \%)$ & \\
\hline Lateral & $69(95.8 \%)$ & $69(95.8 \%)$ & \\
\hline Duration of clamping $(\min )(n=71)^{\mathrm{b}}$ & $22 \pm 8$ & $22 \pm 6$ & 0.848 \\
\hline \multicolumn{4}{|l|}{ Before clamping ${ }^{b}$} \\
\hline Mean BIS & $50 \pm 7$ & $51 \pm 7$ & 0.348 \\
\hline Mean $\mathrm{Cl}\left(\mathrm{L} / \mathrm{min} / \mathrm{m}^{2}\right)$ & $2.7(2.3,3.1)$ & - & - \\
\hline Mean SW (\%) & $6(5,8)$ & - & - \\
\hline Mean SBP $(\mathrm{mmHg})$ & $121 \pm 15$ & $120 \pm 16$ & 0.833 \\
\hline Mean MAP (mmHg) & $88 \pm 10$ & $86 \pm 9$ & 0.181 \\
\hline Mean HR (beats per minute) & $62 \pm 8$ & $60 \pm 9$ & 0.362 \\
\hline \multicolumn{4}{|l|}{ After reperfusion ${ }^{b}$} \\
\hline Mean BIS & $53 \pm 8$ & $51 \pm 7$ & 0.134 \\
\hline Mean $\mathrm{Cl}\left(\mathrm{L} / \mathrm{min} / \mathrm{m}^{2}\right)$ & $3.3(3,3.5) \neq$ & - & - \\
\hline Mean SW (\%) & $5(4,6) \neq$ & - & - \\
\hline Mean SBP $(\mathrm{mmHg})$ & $136 \pm 9$ & $120 \pm 15$ & $<0.001$ \\
\hline Mean MAP (mmHg) & $100 \pm 4$ & $86 \pm 10$ & $<0.001$ \\
\hline
\end{tabular}


Table 2 Intra-/postoperative variables (Continued)

\begin{tabular}{llll}
\hline & Intervention group $(\boldsymbol{n}=\mathbf{7 2})$ & Control group $(\boldsymbol{n}=\mathbf{7 2})$ & $\boldsymbol{P}$ value \\
\hline Mean HR (beats per minute) & $70 \pm 10$ & $63 \pm 8$ & $<\mathbf{0 . 0 0 1}$ \\
Duration of surgery (min) & $93 \pm 38$ & $96 \pm 45$ & 0.629 \\
Use of diuretics within 3 day after surgeryc $^{c}$ & $2(2.8 \%)$ & $1(1.4 \%)$ & 0.560 \\
\hline
\end{tabular}

Data are mean $\pm S D$, number $(\%)$, or median (interquartile range)

$S B P$ systolic blood pressure, MAP mean arterial pressure, $H R$ heart rate, $B I S$ bispectral index, $C l$ cardiac index, SWV stroke volume variation

${ }^{a}$ Only crystalloid fluid was allowed except blood transfusion

bone patient in the intervention group did not undergo renal artery clamping; one in the control group converted to radical nephrectomy without renal artery clamping

$\neq P<0.001$ compared with value before clamping within the intervention group (analyzed with Wilcoxon signed-rank test)

Including torasemide and furosemide

sufentanil $(1.25 \mu \mathrm{g} / \mathrm{ml})$ or morphine $(0.5 \mathrm{mg} / \mathrm{ml})$ and programmed to administer $2-\mathrm{ml}$ boluses with a lockout interval of 6-8 $\mathrm{min}$ and a background infusion rate at 1 $\mathrm{ml} / \mathrm{h}$. Patients were encouraged to eat and drink the day of surgery. Diuretics were provided when considered necessary.

\section{Outcome assessment}

Patients were followed up daily during the first 3 postoperative days, then weekly until 30 days after surgery, and at 3 and 6 months after surgery. Telephone interview was performed for patients after hospital discharge (Supplementary File 1). The primary endpoint was the incidence of AKI within the first 3 postoperative days. The occurrence of AKI was diagnosed according to the serum creatinine level based on the Kidney Disease: Improving Global Outcomes (KDIGO) definition [21], i.e., an increase in serum creatinine by $>0.3 \mathrm{mg} / \mathrm{dl}$ (> $26.5 \mu \mathrm{mol} / \mathrm{l}$ ) within $48 \mathrm{~h}$, or an increase in serum creatinine to $>1.5$ times from baseline within 3 days.

The secondary endpoints included stages of AKI, postoperative length of hospital stay, postoperative complications within 30 days, and 30-day and 6-month mortality. The stage of AKI was classified according to the KDIGO criteria: stage 1 , serum creatinine 1.5-1.9 times baseline or increase by $\geq 0.3 \mathrm{mg} / \mathrm{dl}$ within $48 \mathrm{~h}$; stage 2 , serum creatinine 2-2.9 times baseline; and stage 3 , serum creatinine 3 times baseline or $\geq 4.0 \mathrm{mg} / \mathrm{dl}(\geq 353.6 \mu \mathrm{mol} / \mathrm{l})$

Table 3 Efficacy outcomes

\begin{tabular}{|c|c|c|c|c|}
\hline & $\begin{array}{l}\text { Intervention group }(n= \\
72)\end{array}$ & $\begin{array}{l}\text { Control group }(n= \\
72)\end{array}$ & $\begin{array}{l}\text { Relative risk or median difference }(95 \% \\
\mathrm{Cl}^{\mathrm{a}}\end{array}$ & $P$ value \\
\hline \multicolumn{5}{|l|}{ Primary endpoint } \\
\hline Acute kidney injury (ITT analysis) & $9(12.5 \%)$ & $15(20.8 \%)$ & $\mathrm{RR}=0.60(0.28-1.28)$ & 0.180 \\
\hline Acute kidney injury (PP analysis) & $9(12.7 \%)(n=71)$ & $15(21.1 \%)(n=71)$ & $\mathrm{RR}=0.60(0.28-1.28)$ & 0.179 \\
\hline \multicolumn{5}{|l|}{ Secondary endpoints } \\
\hline Acute kidney injury classification & & & & 0.283 \\
\hline None & $63(87.5 \%)$ & $57(79.2 \%)$ & & \\
\hline Stage 1 & $9(12.5 \%)$ & $12(16.7 \%)$ & & \\
\hline Stage 2 & $0(0.0 \%)$ & $2(2.8 \%)$ & & \\
\hline Stage 3 & $0(0.0 \%)$ & $1(1.3 \%)$ & & \\
\hline Complications within 30 days & $4(5.6 \%)$ & $5(6.9 \%)$ & $\mathrm{RR}=0.80(0.22-2.86)$ & $>0.999$ \\
\hline Postoperative bleeding ${ }^{b}$ & $2(2.8 \%)$ & $1(1.4 \%)$ & & \\
\hline Acute coronary syndrome ${ }^{c}$ & $1(1.4 \%)$ & $0(0.0 \%)$ & & \\
\hline Surgical infection ${ }^{d}$ & $1(1.4 \%)$ & $4(5.6 \%)$ & & \\
\hline $\begin{array}{l}\text { Length of hospital stay after surgery } \\
\text { (day) }\end{array}$ & $4(4,5)$ & $4(4,5)$ & $\mathrm{MD}=0.0(0.0-0.0)$ & 0.541 \\
\hline 30-day mortality & $0(0.0 \%)$ & $0(0.0 \%)$ & - & $>0.999$ \\
\hline 6-month mortality & $0(0.0 \%)$ & $1(1.4 \%)$ & - & $>0.999$ \\
\hline
\end{tabular}

Data are number (\%), mean \pm SD, or median (interquartile range)

$I T T$ intention-to treat, $P P$ per-protocol

${ }^{\text {a } C a l c u l a t e d ~ a s ~ t h e ~ i n t e r v e n t i o n ~ g r o u p ~ v s . ~ o r ~ m i n u s ~ t h e ~ c o n t r o l ~ g r o u p ~}$

${ }^{b}$ Continued decrease of hemoglobin level and required blood transfusion and/or transarterial embolization

'Non-ST elevation myocardial infarction, diagnosed according to serum cardiac troponin I elevation and echocardiographic examination

${ }^{d}$ Fever $>38^{\circ} \mathrm{C}$, increased white blood cell count $\left(>12 \times 10^{9} / \mathrm{L}\right)$ and elevated inflammatory biomarkers necessitating upgrading antibiotic treatment 
or initiation of renal replacement therapy [21]. An exploratory endpoint was estimated glomerular filtration rate (eGFR) on postoperative days 1, 2, and 3 which was calculated with the Chronic Kidney Disease Epidemiology Collaboration (CKD-EPI) equation [22].

\section{Statistical analysis}

\section{Sample size calculation}

Previous studies showed that AKI developed in $42 \%$ of patients after partial nephrectomy [5]. In patients recovering from major abdominal surgery, the incidence of AKI was decreased by up to $60 \%$ with optimized hemodynamic strategy $[11,13]$. We assumed that the incidence of AKI following partial nephrectomy would be reduced to $20 \%$ in patients with goal-directed hemodynamic management, i.e., a $52 \%$ reduction. With significance level set at 0.05 and power set at $80 \%, 68$ patients in each group was required. We planned to enroll 72 patients per group to allow for a $5 \%$ dropout rate.

\section{Outcome analysis}

Baseline balance was assessed with absolute standardized difference, calculated as the absolute difference in means, medians, or proportions divided by the pooled standard deviation [23]. Baseline variables with an absolute standardized difference $\geq 0.327 \quad$ (i.e., 1.96 $\times \sqrt{(\mathrm{n} 1+\mathrm{n} 2) /(\mathrm{n} 1 \times \mathrm{n} 2)})$ were considered imbalanced and would be adjusted in all analyses when considered necessary.

The primary outcome, i.e., the incidence of AKI within 3 days after surgery, was compared with Chi-square tests, with differences between groups expressed as relative risk (95\% CI). Other numeric variables were analyzed using the independent $\mathrm{t}$ test (data with normal distribution) or Mann-Whitney U test (data with nonnormal distribution). Categorical variables were evaluated using the Chi-square test or Fisher's exact test. Repeatedly measured variables like eGFR change over time between groups were analyzed by two-factor repeated measures ANOVA. Repeated measured hemodynamic variables (CI and SVV before and after reperfusion) within the same group were analyzed by Wilcoxon signed-rank test. A two-sided $P<0.05$ was considered statistically significant. Analyses were performed in the intention-to-treat population. Per-protocol analysis was also performed for the primary endpoint. All analyses were performed using SPSS 25.0 software package (IBM SPSS, Chicago, IL).

\section{Results}

From June 16, 2016 to January 2, 2017, 264 patients were screened for study participation. Of these, 144 patients were enrolled into the study and randomly assigned to either the intervention group $(n=72)$ or the control group $(n=72)$. All patients were analyzed according to the intention-to-treat principle. One patient in the intervention group did not undergo renal hilum clamping; one patient in the control group converted to radical nephrectomy. They were excluded from perprotocol analysis for the primary outcome (Fig. 1). Overall, baseline variables were well balanced between two groups except that baseline systolic blood pressure (SBP) was lower in the intervention group than in the control group (Table 1).

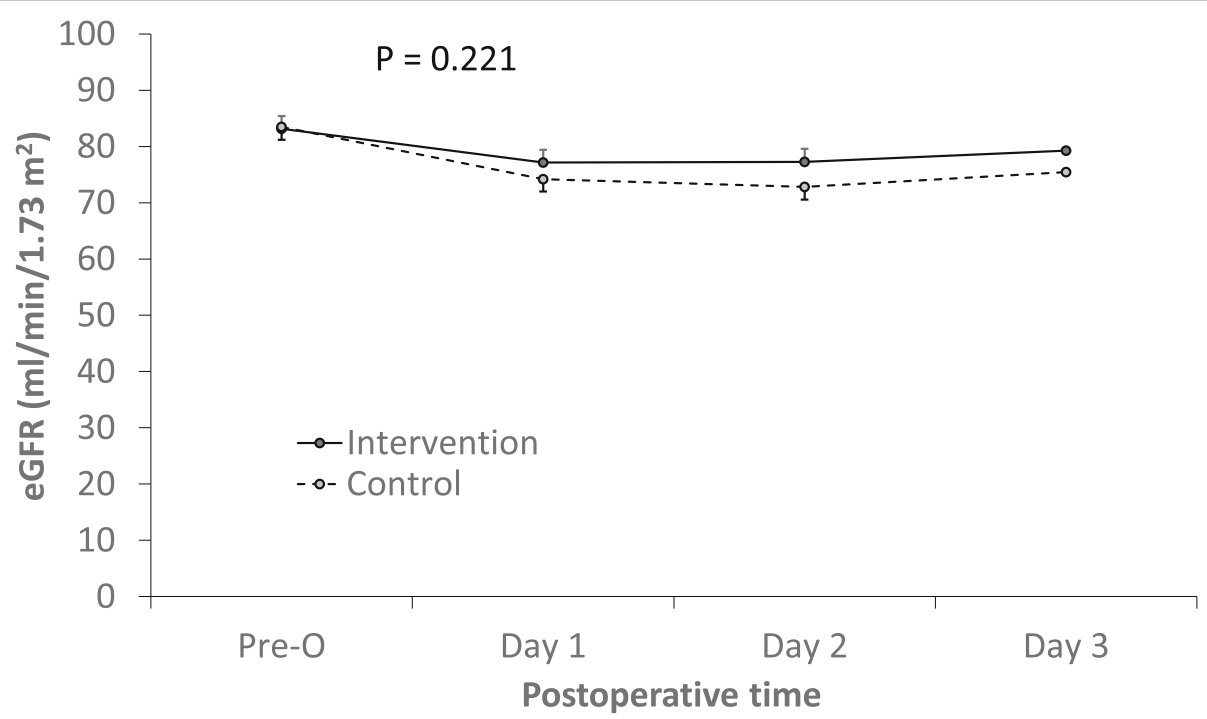

Fig. 2 eGFR changes over time between groups. $P=0.221$ (two-factor repeated measures ANOVA). eGFR, estimated glomerular filtration; calculated according to the Chronic Kidney Disease Epidemiology Collaboration (CKD-EPI) equation [22] 
As expected, patients in the intervention group were given more dobutamine $(P<0.001)$ and norepinephrine $(P=0.028)$ during surgery when compared with the control group; they received more intraoperative fluid infusion $(P=0.012)$ and gave more urine output $(P=0.020)$. After reperfusion, SBP and MAP were higher, and heart rate (HR) was faster in the intervention group than in the control group (all $P<0.001$ ). Other intraoperative variables were comparable between the two groups. For patients in the intervention group, the mean CI was higher whereas the mean SVV was lower after reperfusion than that before clamping (both $\mathrm{P}<0.001$ ) (Table 2 ).

AKI developed in $12.5 \%(9 / 72)$ of patients in the intervention group and in $20.8 \%(15 / 72)$ of patients in the control group; the relative reduction of AKI was $39.9 \%$ in the intervention group but difference was not statistically significant (relative risk [RR] 0.60, 95\% CI 0.281.28; $P=0.180)$. Per-protocol analysis also showed no significant difference between groups $(12.7 \%$ [9/71] vs. $21.1 \%$ [15/71], RR 0.60, 95\% CI 0.28-1.28; $P=0.179$ ) (Table 3).

Regarding secondary endpoints, there were no significant differences in AKI classification, incidence of postoperative 30-day complications, postoperative length of hospital stay, as well as 30-day and 6-month mortality between groups (Table 3 ). None of patient who developed AKI received renal replacement therapy during the postoperative follow-up period. No significant difference was seen in eGFR change over time between two groups (Fig. 2). Safety outcomes from anesthesia induction to $2 \mathrm{~h}$ after surgery did not differ between two groups (Table 4).

\section{Discussion}

Results of this pilot trial showed that, for patients undergoing partial nephrectomy for renal cancer, goaldirected fluid and blood pressure management reduced AKI by about 40\%. However, the trial was underpowered. Large randomized controlled trials are required to confirm our results.

In the present study, AKI occurred in $20.8 \%$ of control group patients. This was lower than we expected [5], but was still within the reported range [5-8, 24, 25]. Two reasons might explain the unexpected lower incidence of AKI in our control group patients. The first one is the diagnostic criteria. AKI is usually diagnosed within 7 postoperative days according to the KDIGO criteria. In the present study, the majority of our patients were discharged within 3 to 4 days after partial nephrectomy. Furthermore, diuretics were commonly used during the perioperative period, these made urine output an unreliable parameter for AKI diagnosis; and early urine catheter removal made it difficult to monitor urine output per hour in the ward. Therefore, we diagnosed AKI only according to serum creatinine change within 3 postoperative days. This might have underestimated the rate of AKI development. However, recent studies also showed that the majority of surgery-related acute kidney injury occurred within $48 \mathrm{~h}$ of surgery [26]. Secondly, the improvement of surgeons' skill and surgical technique helped preserve renal function. For example, the durations of renal hilus clamping and surgery were shorter in our patients than in previous studies [8, 24, 25].

Routine circulatory management during partial nephrectomy is to maintain blood pressure change within $20 \%$ from baseline and urine output $>0.5 \mathrm{ml} / \mathrm{kg} / \mathrm{h}$.

Table 4 Safety outcomes ${ }^{a}$

\begin{tabular}{|c|c|c|c|}
\hline & Intervention group $(n=72)$ & Control group $(n=72)$ & $P$ value \\
\hline Hypertension $^{b}$ & $19(26.4 \%)$ & $11(15.3 \%)$ & 0.101 \\
\hline Hypotension ${ }^{c}$ & $11(15.3 \%)$ & $19(26.4 \%)$ & 0.101 \\
\hline Tachycardia $^{d}$ & $7(9.7 \%)$ & $4(5.6 \%)$ & 0.347 \\
\hline Bradycardia $^{e}$ & $26(36.1 \%)$ & $30(41.7 \%)$ & 0.494 \\
\hline Frequent ventricular premature beat ${ }^{f}$ & $0(0.0 \%)$ & $1(1.4 \%)$ & $>0.999$ \\
\hline Massive bleeding ${ }^{9}$ & $0(0.0 \%)$ & $2(2.8 \%)$ & 0.497 \\
\hline Airway spasm ${ }^{\mathrm{h}}$ & $2(2.8 \%)$ & $1(1.4 \%)$ & $>0.999$ \\
\hline Respiratory alkalosis ${ }^{i}$ & $0(0.0 \%)$ & $1(1.4 \%)$ & $>0.999$ \\
\hline
\end{tabular}

Data are presented as number of patients (percentage)

${ }^{a}$ Safety outcome were monitored from anesthesia induction to $2 \mathrm{~h}$ after surgery

bystolic blood pressure $>180 \mathrm{mmHg}$ or an increase of more than $30 \%$ from baseline

'Systolic blood pressure $<90 \mathrm{mmHg}$ or a decrease of more than $30 \%$ from baseline

${ }^{d}$ Heart rate $>100 \mathrm{bpm}$ or an increase of more than $30 \%$ from baseline

${ }^{\mathrm{e}} \mathrm{Heart}$ rate $<50 \mathrm{bpm}$ or a decrease of more than $30 \%$ from baseline

${ }^{f} \mathrm{New}$-onset ventricular premature beat that required antiarrhythmic therapy

${ }^{9}$ Volume of intraoperative bleeding $>1000 \mathrm{ml}$

${ }^{h}$ Wheezing rales heard in the lung field, with or without decrease of $\mathrm{SpO}_{2}$; relieved after intravenous corticosteroids or aminophylline

'Diagnosed according to blood gas results, occurred within $1 \mathrm{~h}$ after surgery in the ward and relieved after mask oxygen inhalation 
However, the incidence of AKI remains high after surgery $[8,24,25]$. Experience from kidney transplantation suggested that maintaining adequate renal hydration and higher blood pressure after reperfusion (i.e., CVP $>8$ $\mathrm{mmHg}$ and MAP $>95 \mathrm{mmHg}$ ) are beneficial for graft function $[14,15,18]$. Similar hemodynamic therapy may also relieve ischemia-reperfusion injury and protect renal function after partial nephrectomy.

Kidney is more sensitive to inadequate hydration compared with other organs. As Myles et al. [27] reported, restrictive fluid therapy is associated with a higher risk of AKI in renal transplant recipients. Static cardiac filling pressures such as CVP correlate poorly with the intravascular volume [28]; and hydration according to static parameters may induce excessive fluid infusion [29]. Better hemodynamic monitoring can be achieved with $\mathrm{LiDCO}^{\mathrm{ra}-}$ pid, a minimal invasive device that can monitor SVV, cardiac output and cardiac index through pressure contour analysis [30]. As a dynamic parameter, SVV is capable to reflect volume responsiveness and replace CVP [19, 28]. It was found that the optimal cutoff value of SVV is $6 \%$ and can be used as an alternative to CVP of $8 \mathrm{mmHg}$ during kidney transplantation [19]. Therefore, SVV was maintained $<6 \%$ in this pilot trial as a hydration goal.

Cardiac output is an indicator of oxygen delivery and organ perfusion but is often compromised during general anesthesia. Studies showed that low-dose inotropic therapy is associated with an improved global oxygen delivery and tissue oxygenation [11]. However, in the study of Pearse et al. [12], cardiac-output guided hemodynamic management did not reduce complications including AKI after major gastrointestinal surgery. To be noted, dopexamine, a $\beta 2$-agonist with both inotropic and vasodilator effects, was infused to obtain cardiac inotropy in the above study; blood pressure was ignored and might even be lower than usual due to the vasodilator effects of dopexamine, and as a result renal perfusion pressure was not guaranteed. In clinical practice, dopamine is also frequently used to increase blood pressure during kidney transplantation. But studies indicate that dopamine does not improve kidney function; on the contrary, it may produce potential harmful effects [31]. In this pilot study, dobutamine was adopted to maintain normal cardiac output and MAP $>95 \mathrm{mmHg}$ in the intervention group; norepinephrine was infused if necessary.

This pilot study was the first to explore the effect of goal-directed circulatory management on renal function after partial nephrectomy. It seems that circulatory management with the goals of $\mathrm{SVV}<6 \%$ MAP $>95 \mathrm{mmHg}$ and CI $3.0-4.0 \mathrm{~L} / \mathrm{min} / \mathrm{m}^{2}$ based on $\mathrm{LiDCO}{ }^{\text {rapid }}$ hemodynamic monitoring didn't significantly reduce postoperative AKI when compared with routine circulatory management, very possibly due to under-powered sample size. However, the relative risk reduction of AKI approaches $40 \%$, which cannot be ignored and is clinically important. Our trial was underpowered because AKI incidence was lower than expected, and intervention reduced AKI by $40 \%$ rather than anticipated $52 \%$. With the baseline AKI incidence of $20.8 \%$ and treatment effect of $40 \%, 626$ patients would be required to provide $80 \%$ power. Further studies with larger sample sizes are needed to confirm our results.

Our study confirmed that patients' overall renal function declined after surgery and, of those who developed AKI, most had mild renal injury. Our results were similar to previous studies [8]. Severe AKI is associated with increased mortality [32]; furthermore, mild AKI also negatively affected long-term functional recovery after partial nephrectomy and may increase the proportion of CKD upstaging $[33,34]$.

There are some limitations in this trial. Firstly, as a single-center study, the generalizability of our results may be limited. Secondly, interventions could not be blinded to anesthesiologists taking care of patients, which may bring bias. To reduce the related bias, anesthesiologists did not participate in patient recruitment and postoperative follow-up; whereas investigators who performed follow-ups were masked from study group assignment. Thirdly, AKI was diagnosed only according to serum creatinine level. This might have underestimated the real rate of AKI. Lastly, as a pilot study, the limited sample size diminished study power.

\section{Conclusions}

For patients undergoing partial nephrectomy, goaldirected circulatory management to maintain $\mathrm{SVV}<6 \%$, MAP > $95 \mathrm{mmHg}$ and CI $3.0-4.0 \mathrm{~L} / \mathrm{min} / \mathrm{m}^{2}$ from renal artery clamping to the end of surgery reduced postoperative AKI by $40 \%$, although not significantly so. Further studies with larger sample sizes are required.

\section{Abbreviations}

AKI: Acute kidney injury; IRI: Ischemia-reperfusion injury; CVP: Central venous pressure; MAP: Mean arterial pressure; eGFR: estimated glomerular filtration rate; PADUA score: Preoperative aspects and dimensions used for an anatomical score; SW: Stroke volume variation; Cl: Cardiac index; KDIGO: the Kidney Disease: Improving Global Outcomes; CKD-EPI: the Chronic Kidney Disease Epidemiology Collaboration; HR: Heart rate; ASD: Absolute standardized difference; SBP: Systolic blood pressure; ITT: Intention-to-treat; PP: Per-protocol

\section{Supplementary Information}

The online version contains supplementary material available at https://doi. org/10.1186/s12871-021-01288-8.

\section{Additional file 1 .}

\section{Acknowledgements}

The authors thank Xue-Ying Li (Department of Biostatistics, Peking University First Hospital, Beijing, China) for statistical analysis. 


\section{Authors' contributions}

DXW, HK and QFW designed this study. QFW analyzed the data and drafted the manuscript. DXW and DLM critically revised the manuscript. QFW, HK, ZZX, HJL and DLM participated in the conduct of the study. All authors read and approved the final manuscript.

\section{Funding}

None.

\section{Availability of data and materials}

The datasets used and/or analyzed during the current study are available from the corresponding author on reasonable request.

\section{Declarations}

\section{Ethics approval and consent to participate}

The study was approved by the Clinical Research Ethics Committee of Peking University First Hospital (2016[1118]) on May 3, 2016. Written informed consent to participate was obtained from all patients.

\section{Consent for publication}

Not applicable.

\section{Competing interests}

The authors declare that they have no competing interests.

Received: 27 September 2020 Accepted: 24 February 2021

Published online: 03 March 2021

\section{References}

1. Uzzo R, Novick A. Nephron sparing surgery for renal tumors: indications, techniques and outcomes. J Urol. 2001;166(1):6-18.

2. Lane B, Russo P, Uzzo R, et al. Comparison of cold and warm ischemia during partial nephrectomy in 660 solitary kidneys reveals predominant role of nonmodifiable factors in determining ultimate renal function. J Urol. 2011;185(2):421-7.

3. Kotamarti S, Rothberg M, Danzig M, et al. Increasing volume of nonneoplastic parenchyma in partial nephrectomy specimens is associated with chronic kidney disease upstaging. Clin Genitourin Cancer. 2015;13(3):239-43.

4. Thompson R, Lane B, Lohse $C$, et al. Every minute counts when the renal hilum is clamped during partial nephrectomy. Eur Urol. 2010;58(3):340-5.

5. Zabell J, Isharwal S, Dong W, et al. Acute kidney injury after partial nephrectomy of solitary kidneys: impact on long-term stability of renal function. J Urol. 2018;200(6):1295-301.

6. Yoon HK, Lee HJ, Yoo S, et al. Acute kidney injury adjusted for parenchymal mass reduction and long-term renal function after partial nephrectomy. J Clin Med. 2019;8(9):1482.

7. Zhang Z, Zhao J, Dong W, et al. Acute kidney injury after partial nephrectomy: role of parenchymal mass reduction and ischemia and impact on subsequent functional recovery. Eur Urol. 2016;69(4):745-52.

8. Rajan S, Babazade R, Govindarajan S, et al. Perioperative factors associated with acute kidney injury after partial nephrectomy. Br J Anaesth. 2016; 116(1):70-6.

9. Chawla LS, Eggers PW, Star RA, et al. Acute kidney injury and chronic kidney disease as interconnected syndromes. N Engl J Med. 2014;371(1):58-66.

10. Mehta S, Chauhan K, Patel A, et al. The prognostic importance of duration of AKl: a systematic review and meta-analysis. BMC Nephrol. 2018;19(1):91.

11. Jhanji S, Vivian-Smith A, Lucena-Amaro S, et al. Haemodynamic optimisation improves tissue microvascular flow and oxygenation after major surgery: a randomised controlled trial. Crit Care. 2010;14(4):R151.

12. Pearse $\mathrm{R}$, Harrison $\mathrm{D}, \mathrm{MacD}$ nald $\mathrm{N}$, et al. Effect of a perioperative, cardiac output-guided hemodynamic therapy algorithm on outcomes following major gastrointestinal surgery: a randomized clinical trial and systematic review. JAMA. 2014:311(21):2181-90.

13. Futier $E$, Lefrant J, Guinot $P$, et al. Effect of individualized vs standard blood pressure management strategies on postoperative organ dysfunction among high-risk patients undergoing major surgery: a randomized clinical trial. JAMA. 2017;318(14):1346-57.

14. Bacchi G, Buscaroli A, Fusari M, et al. The influence of intraoperative central venous pressure on delayed graft function in renal transplantation: a singlecenter experience. Transplant Proc. 2010;42(9):3387-91.
15. Aulakh N, Garg K, Bose A, et al. Influence of hemodynamics and intraoperative hydration on biochemical outcome of renal transplant recipients. J Anaesthesiol Clin Pharmacol. 2015;31(2):174-9.

16. Tóth $\mathrm{M}$, Réti $\mathrm{V}$, Gondos $\mathrm{T}$. Effect of recipients' peri-operative parameters on the outcome of kidney transplantation. Clin Transpl. 1998;12(6):511-7.

17. Campos L, Parada B, Furriel F, et al. Do intraoperative hemodynamic factors of the recipient influence renal graft function? Transplant Proc. 2012;44(6): 1800-3.

18. Calixto Fernandes M, Schricker T, Magder S, et al. Perioperative fluid management in kidney transplantation: a black box. Crit Care. 2018;22(1):14.

19. Chin J, Jun I, Lee J, et al. Can stroke volume variation be an alternative to central venous pressure in patients undergoing kidney transplantation? Transplant Proc. 2014;46(10):3363-6.

20. Ficarra V, Novara G, Secco S, et al. Preoperative aspects and dimensions used for an anatomical (PADUA) classification of renal tumours in patients who are candidates for nephron-sparing surgery. Eur Urol. 2009;56(5):786-93.

21. Khwaja A. KDIGO clinical practice guidelines for acute kidney injury. Nephron Clin Pract. 2012;120(4):c179-84.

22. Levey A, Stevens L, Schmid C, et al. A new equation to estimate glomerular filtration rate. Ann Intern Med. 2009;150(9):604-12.

23. Ali M, Groenwold R, Pestman W, et al. Propensity score balance measures in pharmacoepidemiology: a simulation study. Pharmacoepidemiol Drug Saf. 2014:23(8):802-11.

24. Nativ O, Bahouth Z, Sabo E, et al. Method used for tumor bed closure (suture vs. sealant), ischemia time and duration of surgery are independent predictors of post-nephron sparing surgery acute kidney injury. Urol Int. 2018;101(2):184-9.

25. Chen J, Lin J, Lin C. Serum and urinary biomarkers for predicting acute kidney injury after partial nephrectomy. Clin Invest Med. 2015;38(3):E82-9.

26. Li S, Wang S, Priyanka $P$, et al. Acute kidney injury in critically ill patients after noncardiac major surgery: early versus late onset. Crit Care Med. 2019; 47(6):e437-e44.

27. Myles $\mathrm{P}$, Bellomo R, Corcoran T, et al. Restrictive versus Liberal fluid therapy for major abdominal surgery. N Engl J Med. 2018;378(24):2263-74.

28. Toyoda D, Fukuda M, Iwasaki R, et al. The comparison between stroke volume variation and filling pressure as an estimate of right ventricular preload in patients undergoing renal transplantation. J Anesth. 2015;29(1): $40-6$.

29. Holte K, Sharrock N, Kehlet H. Pathophysiology and clinical implications of perioperative fluid excess. Br J Anaesth. 2002;89(4):622-32.

30. O'Loughlin E, Ward M, Crossley A, et al. Evaluation of the utility of the Vigileo FloTrac $\left.{ }^{(T M}\right)$, LiDCO(TM) , USCOM and CardioQ $\left.{ }^{(T M}\right)$ to detect hypovolaemia in conscious volunteers: a proof of concept study. Anaesthesia. 2015;70(2):142-9.

31. Ciapetti M, di Valvasone S, di Filippo A, et al. Low-dose dopamine in kidney transplantation. Transplant Proc. 2009;41(10):4165-8.

32. Hoste E, Bagshaw $\mathrm{S}$, Bellomo R, et al. Epidemiology of acute kidney injury in critically ill patients: the multinational AKI-EPI study. Intensive Care Med. 2015:41(8):1411-23.

33. Bravi CA, Vertosick E, Benfante N, et al. Impact of acute kidney injury and its duration on long-term renal function after partial nephrectomy. Eur Urol. 2019;76(3):398-403.

34. Turan A, Cohen B, Adegboye J, et al. Mild acute kidney injury after noncardiac surgery is associated with long-term renal dysfunction: a retrospective cohort study. Anesthesiology. 2020;132(5):1053-61.

\section{Publisher's Note}

Springer Nature remains neutral with regard to jurisdictional claims in published maps and institutional affiliations. 